\title{
Planetariums, theatres of the Universe
}

\author{
Agnès Acker ${ }^{1,2}$ \\ ${ }^{1}$ Observatoire Astronomique de Strasbourg, \\ 11, rue de l'Université, 67000 Strasbourg, France \\ email: acker@astro.u-strasbg.fr \\ ${ }^{2}$ Association des Planétariums de Langue Française
}

\begin{abstract}
The history of this magic instrument is complex, and the teaching goal was never forgotten. Technological developments of the planetarium enable it now to treat the major topics of astronomy and astrophysics. But by simplifying too much or badly, one is likely to bring confusion in the spirits. The development of the planetarium is a continual challenge, as long as the scientific culture is not integrated perfectly in the cultural landscape. In several countries, planetariums link their knowledge and their know-how, for better resisting to economic difficulties, and better speaking about astronomy to their public. In particular the great project ALMA will be celebrated in a show for the International Year of Astronomy. Today's planetarium works as a theater for all sciences, and can issue a passport to the various scales of the universe
\end{abstract}

Keywords. Planetarium, Technology, Associations, Digital theater, ALMA

\section{Introduction}

When the night falls, we are charmed by the beauty and the mystery of the starry night. At the same time, a feeling of concern, even of anguish, can seize us. What are these scintillating lights? Why some of them are moving ? Is there a relation between these stars and ourselves? But, now, the night is illuminated by the lights of our cities, the Milky Way becomes unknown, and we loose both contemplation and interrogation. The planetarium, large or small, can help us to recover our cosmic dimension.

\section{The planetarium: a short story}

In the French language, the word planetarium is very ambiguous : sometimes it means the instrument, sometimes it means the place where this device works. More and more, this term refers to the operating structure, called theater in the US. Here we focus on the simulator.

\subsection{The precursors}

The planetariums were constructed to simulate and explain the movement of planets. It seems that Archimedes (about $250 \mathrm{BCE}$ ) reproduced it with a water mechanism. In most antique planetariums, the stars were not present : they had no mysterious journey, they were only bright spots. One of the first planetariums was an Orrery, whose name is an allusion to Charles Boyle, 4th Earl of Orrery in Ireland, who had achieved such a device by John Rowley in 1716. It is a representation of the sky seen from the outside and centred on the Sun, while now planetariums corresponds to the concept of a system seen from the inside, centered on Earth (Fig. 1). Between 1650 and 1664, the German Andreas Bösch in collaboration with the Dutch Adam Olearius built a globe of 3.11 metres in diameter, within which a dozen people could penetrate. This device, which can be truly regarded as the ancestor of our modern planetariums, was given to Tsar 


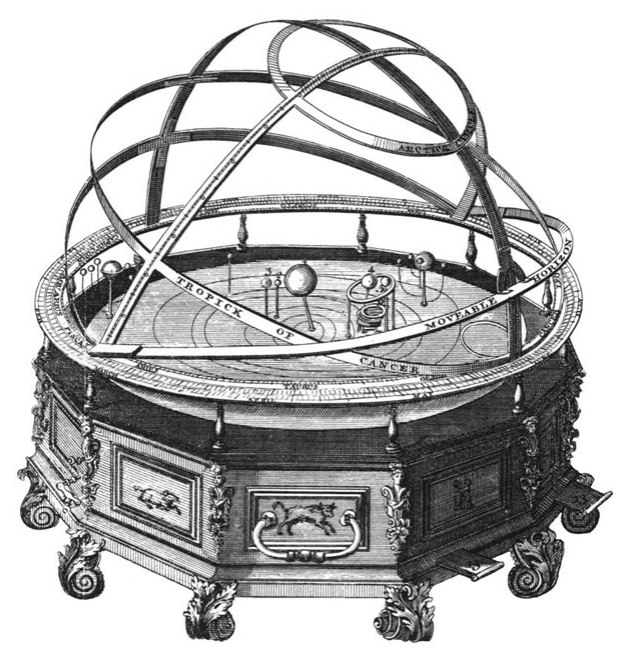

Figure 1. Orrery device (1704).
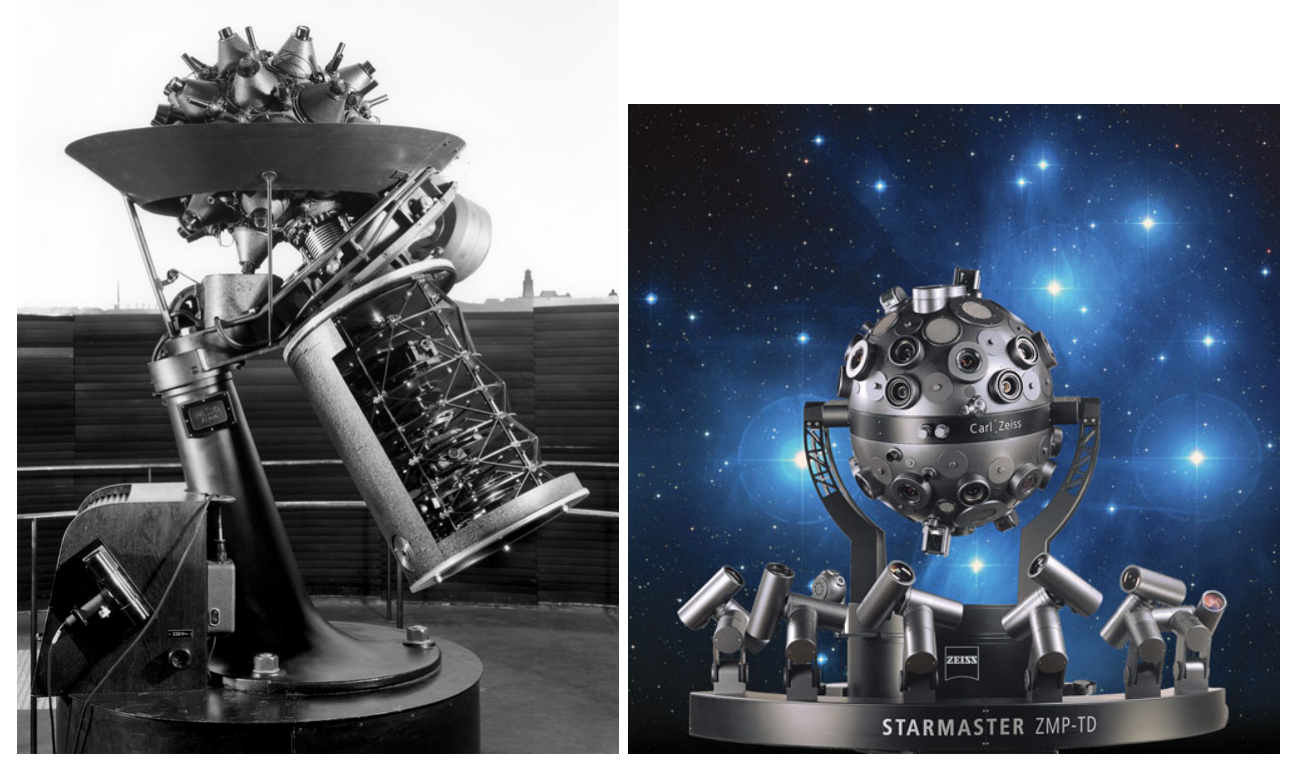

Figure 2. Zeiss planetariums. (Left) prototype 1923 (c) Zeiss). (Right) STARMASTER (1997), Palais de la Découverte, Paris (c) Zeiss).

Peter the Great in 1713 in Saint Petersburg. A copy of the Gottorp globe, but 18 feet in diameter and for 30 people, was conducted in Great Britain for the University of Cambridge. A more modern version of the world Gottorp was built between 1912 and 1913 in the United States, in Chicago (Museum of the Academy of Sciences).

\subsection{Zeiss planetariums}

Since 1913 at the initiative of Professor Max Wolf, former director of the Observatory in Heidelberg, and Oskar von Miller, founder of the Deutsches Museum in Munich, a study was undertaken to find the best method for the artificial representation of the sky. Two planetariums were built for the Deutsches Museum in Munich: one Copernican, by 


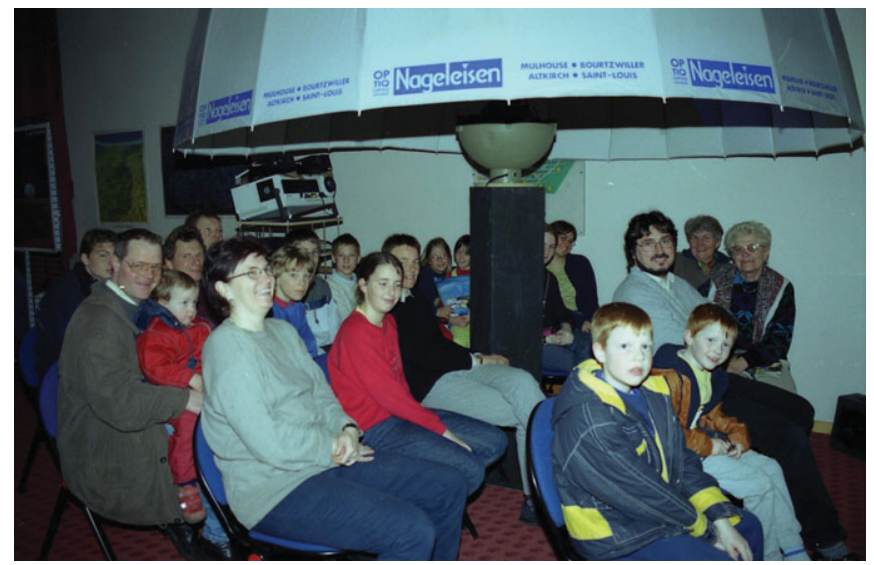

Figure 3. Mobile Planetarium in Kruth, France (c) Lionel Muller.

Franz Meyer, the other Ptolemaic by Walter Bauersfeld. Finally, after much hesitation, the solution of the geocentric planetarium was chosen to recreate in the planetarium the majestic silence of the nature we can contemplate : the Zeiss factory was born (see Fig. 2).

The first French planetarium was built under the 1937 Universal Exhibition in Paris. As the exhibition closed on 11 November 1937, it was dismantled and stored in the basement of the Conservatoire National des Arts et Métiers. Later, the first installation consisted of a dome $22.5 \mathrm{~m}$ in diameter containing 370 seats. The projector was a Zeiss. It was not until the year 1952 for this Planetarium to open its doors to the public, in a final and mythical place: the Palais de la Découverte. The first show presented to the public in 1925 caused great admiration for the Zeiss technological miracle Zeiss Malburet $(2008$.

\subsection{New generation projectors}

Other manufacturers, both American (Spitz) and Japanese (GOTO, Konica-Minolta) have built opto-mecanical projectors inspired by Zeiss. In 1950, the small projectors Spitz 512 enriched more than 512 classrooms in the USA. Then, from 1965, it was the era of multi-image theatres, with immersive projection of allskies and video animations, in harmony with the golden era of the conquest of the Moon, and political interest in a prestigious communication to the public.

\subsection{Mobile planetariums}

The launch the first satellite (Sputnik 1 in 1957) created a craze for all things coming to astronomy : schools, museums, cities wanted to have a planetarium. Given the price and difficulty of creating a good size, a new dynamic was set up with the development of small mobile planetariums. The dome became inflatable, transportable in a car. The projector is simplified in the extreme and should also be transportable (Fig. 3). After the Japanese Goto (early 1970) followed by American Starlab (1977), the French RSA Cosmos develops on a large scale this new generation of planetariums. These small projectors generally have a ball throwing stars, the magnitude of the stars is simulated by stenopes of different diameters with regard to the planets, which are produced by small moving parts drawing manually. 

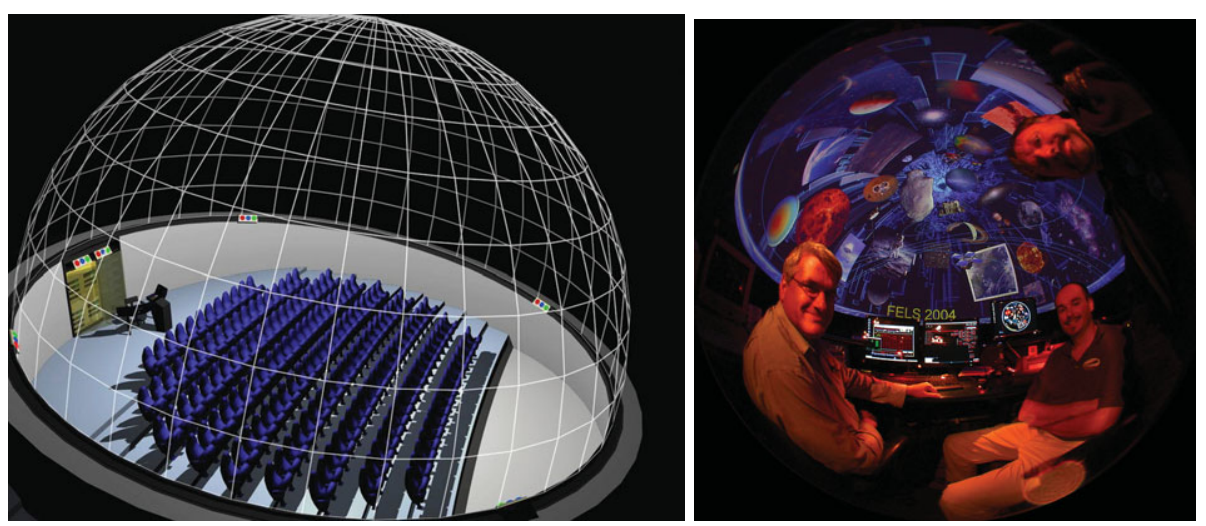

Figure 4. Digital Planetariums. (Left) : Digital dome (c) RSAcosmos. (Right): Fels theater at SkySkan.

\subsection{Digital planetariums}

The advent of computers and technology determined a revolution in the world of planetariums. Since 1980 new devices will emerge : a digital planetarium, which is a powerful computer, where the traditional display is replaced by a fish-eye to project the image on the surface of the dome. The latest versions replace the central projection device by variable number of tritubes video projectors distributed on the periphery of the room. The possibilities of a huge information will now be prevail over the quality of the projected sky.

Evans \& Sutherland, named after its two founders (David Evans and Ivan Sutherland), put on the market in 1983 the first digital planetarium (Digistar), which was drafted in 1979 in Salt Lake City (Utah, USA) (Fig. 12). The U.S.A.-based firm Sky-Skan, founded in 1967 with a view to creating special effects projectors for planetariums, also offers digital models (DigitalSky). The first room equipped by it was the Christa McAuliffe Planetarium in Concord (New Hampshire, U.S.A.) in 1990. The French firm RSA Cosmos proposed in 1988 an opto-mechanical device (SN88, the first model equipped the Planetarium of Cappelle-la-Grande), and began the production of digital devices (InSpace System) (Fig. 4). Manufacturers of digital planetariums will propose, in addition to a computer system and the associated projector, a range of benefits, including shows.

At present, a real struggle for domination of the market for large structures takes place between the giants of the computer manufacturers and more traditional devices. The choice will be made taking into account one of two currently still irreconcilable aspects : the quality of the starry sky, or the opportunities related to computer technology. Some installations are seeking to collect this double aspect : a Zeiss or GOTO optomecanical projector with a digital environment. It will certainly take many years before digital technology comes to the quality of the sky done by an opto-mechanical projector. When this capacity will be reached - perhaps laser technology ? - the two systems could merge for the delight of spectators and the economic benefit of the Planetarium directors.

\subsection{Self-made planetariums}

Recently, we observe many models built by amateurs. A historical meeting for small numerical planetariums was hold in december 2008 under the $6 \mathrm{~m}$ dome of the planetarium from Marseille - the smallest high-technology theatre in Europe. Lionel Ruiz and Yves 


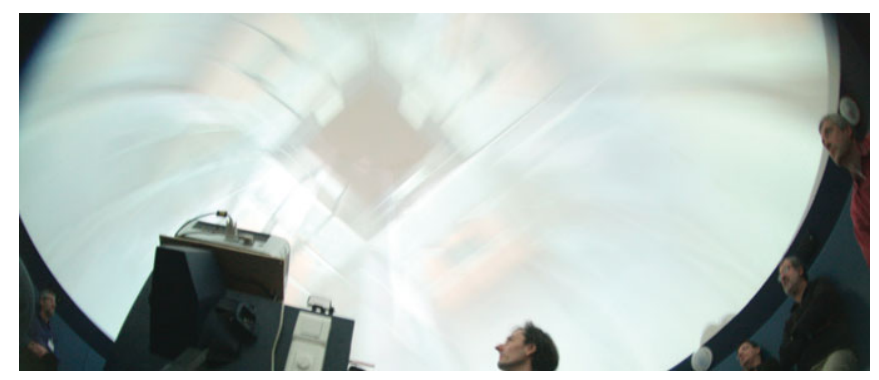

Figure 5. The Lhoumeau-Sky-System (c) Planetarium, Marseilles, 2008

Lhoumeau demonstrated the fantastic possibilities of the software Stellarium for FullDomeVideo projection using the new version of LSS : the Lhoumeau Sky-System is a concept of planetarium at low cost for small and medium theaters (Fig. 5).

\section{The evolution of planetariums}

\subsection{How to resist to economic difficulties?}

To develop while resisting the economic difficulties is a continual challenge, as long as the scientific culture is not integrated perfectly in the cultural landscape. The goals of Planetariums remain constant, while the concerns and priorities of wider society molded and reinterpreted those goals to fit the economic and ideological pressures of the time.

James Croft (2008)

The type of planetarium is chosen according to the economic constraints of the planetarium, in relation with the factory installed in each country : Zeiss projectors equipped $90 \%$ of German theatres during decades. Now, digital planetariums have more and more success around the world (Fig. 6). However, full-dome shows are very expensive to produce Mike (2008). In the words of Sharon Shanks (December 2008), editor of the Planetarian journal: Planetariums are continuing to adopt full-dome but it's obvious the change is being made by those facilities able to scrap the funding together or write creative and compelling grant applications. Note that a return on investment could only be make by licensing a full-dome show to multiple users.

For better alluring their visitors, the large fixed planetariums must diversify their services. To catch a broader market, and complete the scientific message, besides the planetarium presentations, the visitor is invited in an interactive museum and an astronomical shop. Some Planetariums present concerts - as the wonderful show by Christine Högl with her toothing-stone (Fig. 7).

\subsection{Education against entertainment?}

Planetariums lie between education and entertainment. They navigate between these potentially conflicting demands. The Full Dome Video shows immerse the visitor in a virtual world, and proposes travels to the center of the Sun, or to black holes ! The description of the starry sky is replaced by stories and tales, with strange heroes. For better attracting their customers, some show producers choose as narrators well known actors : Harrison Ford, Tom Hanks, etc.

The new technology is sometimes excellent to explain the laws of physics. In the IYAALMA show (In Search of our Cosmic Origins), an astronomer is sitting near a fire : she explains to us that bright yellow flames will be very hot, and over the amber coal while tuning red, and finally dark : a heat is still be felt, index of an invisible light, the infrared. 

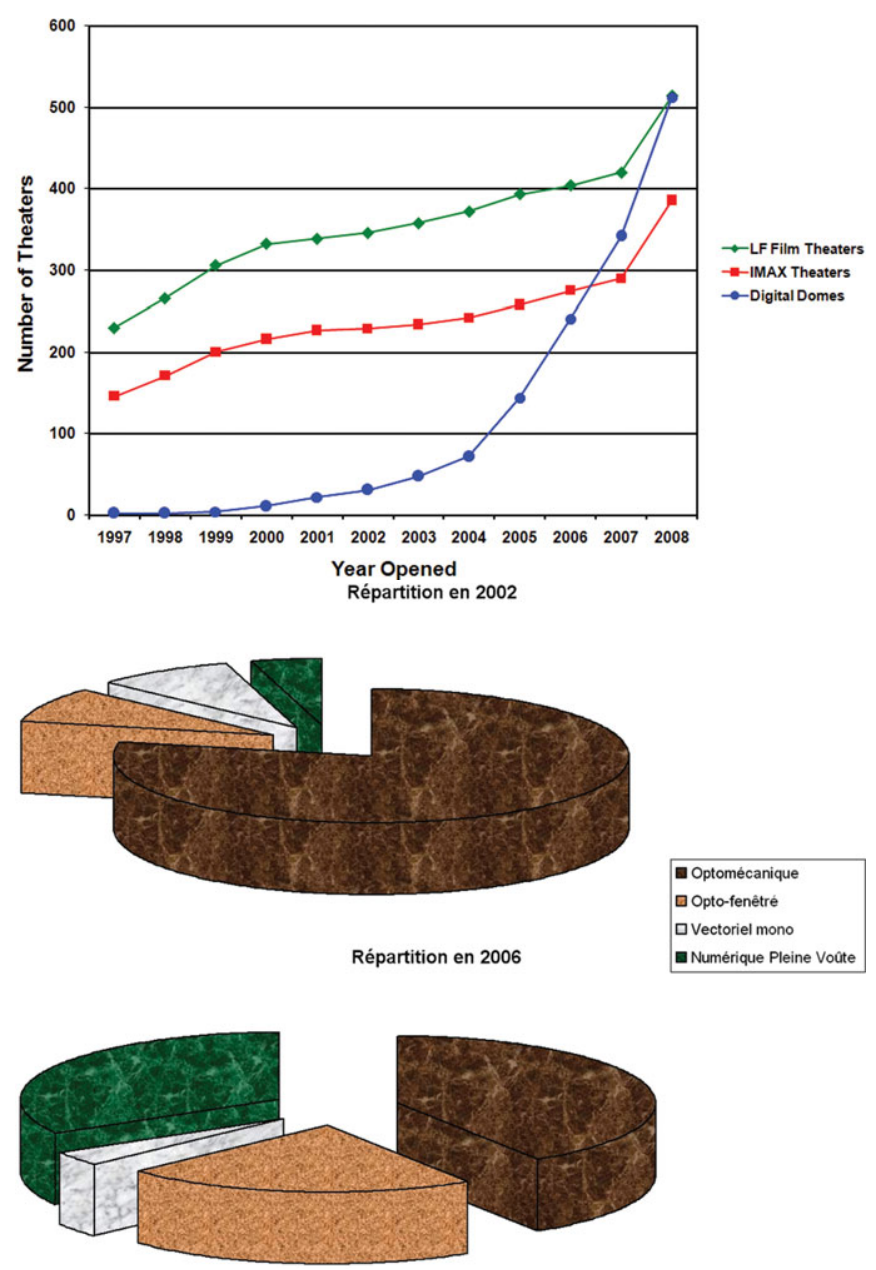

Figure 6. Evolution of planetariums. (Top) Distribution of optomecanical and numerical devices in France in 2002 and 2006 (C) Lionel Ruiz). (Bottom) Comparative evolution of Films, IMAX, and Digital planetariums, see Lantz (2009) (C) Ed Lantz).

And so we see that the color and the temperature are related : cool matter can radiate infrared light, this could be an excellent introduction to multi-wavelength astronomy!

\subsection{Major issues..... and misconceptions}

Digital Planetariums also allow imaginary travel to discover that the Milky Way is a galaxy, by flying through hundreds of light-years in a few minutes. The digital techniques make it possible to simulate all the distances and objects of the universe, and their interactions. But they can also generate confusion in the spirits of the visitors. First of all, one loses the correct perception of the distances in the cosmos. The technique Powers of Ten remains invaluable to recall that Proxima Centauri is 250,000 times more distant from us than the Sun. It seems also necessary to show the very small size on the sky explored by each image of the HST. Other point : the cosmological approaches must respect unimaginable dimensions of space and time, and avoid the words like infinite, creation at moment zero, etc. Cosmology in a planetarium is unable to save the universe in a bag : the big bang is a model, not an explosive event, it implicates an expansion of 


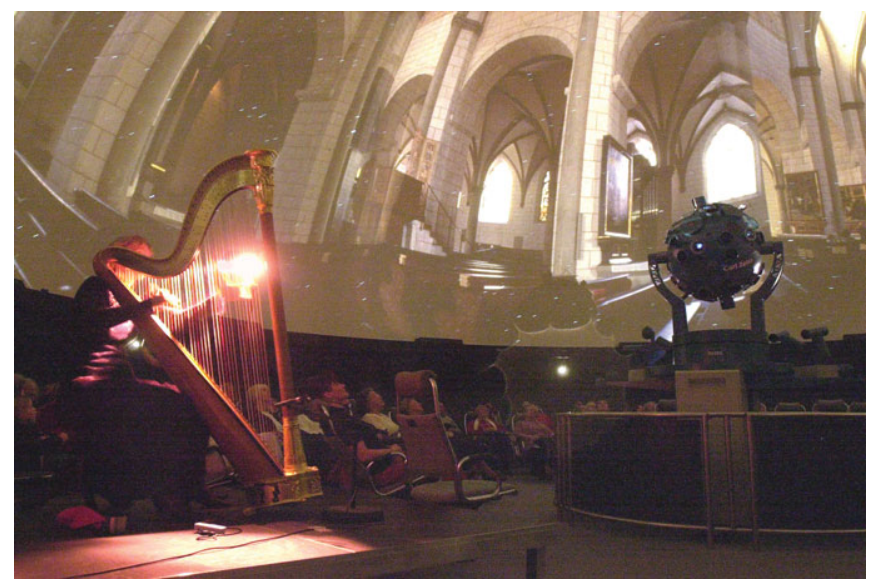

Figure 7. Concert by Christine Högl at the Wolfsburg Zeiss Planetarium (c) C. Högl).

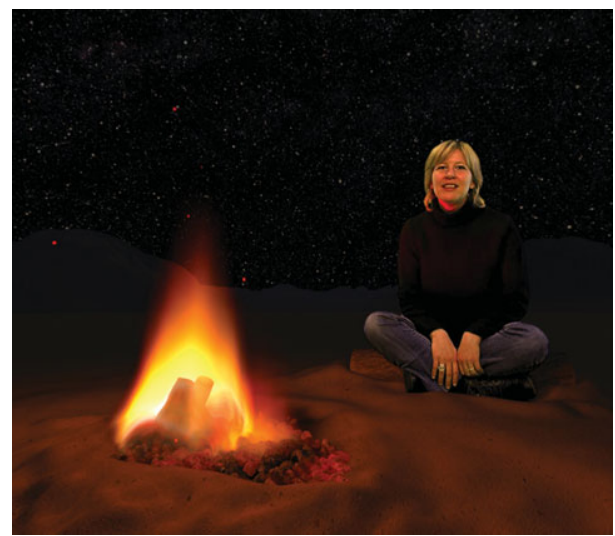

Figure 8. Ariane explains the invisible light in the ALMA show (CAPLF).

space, not an expansion in space, galaxies are co-mobile on the expanding space and their light waves are elongated and reddened more and more when their distance increases.

\section{The Planetarium community}

\subsection{IPS and $A P L F$}

The International Planetarium Society (IPS) founded in 1970 is the global association of planetarium professionals, with nearly 700 members coming from 35 countries around the world. They represent schools, colleges and universities, museums, and public facilities of all sizes including both fixed and portable planetariums. As explained at www.ipsplanetarium.org the primary goal of the Society is to encourage the sharing of ideas among its members through conferences, publications, and networking. IPS membership is open to anyone interested in planetariums : directors, teachers, technicians, writers, artists, media specialists, presenters, vendors, scientists, students, and sponsors and friends of the planetarium dome and its starry sky. More than 20 regional and national planetarium associations from around the world are affiliated. An IPS conference is organised every 

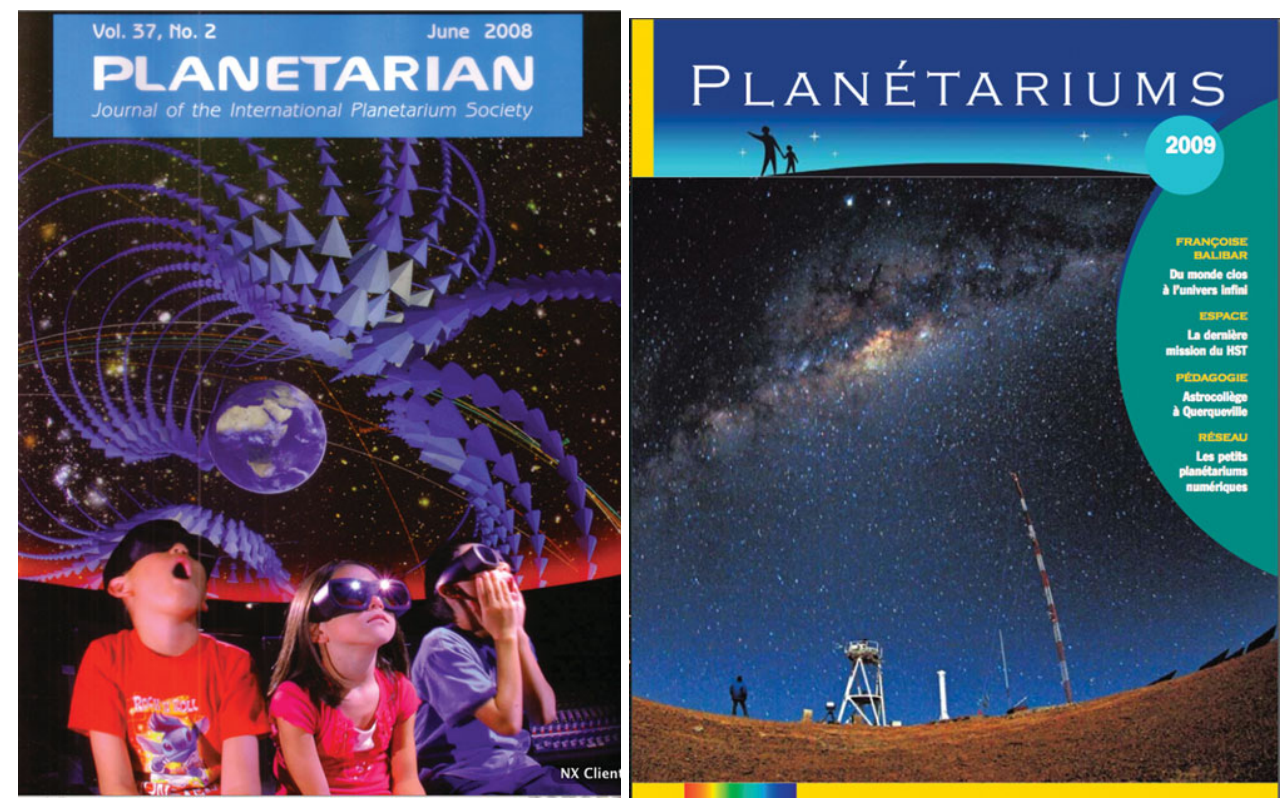

Figure 9. The journals PLANETARIAN of IPS and PLANETARIUMS of APLF.

2 years. The Journal PLANETARIAN is edited three times each year, and its diffusion is accompanied by gifts such as CDROMs and DVDs.

The Association des Planétariums de Langue Franaise was born in 1984 in Strasbourg, and gathers 28 theaters (dome sizes ranging from $6 \mathrm{~m}$ to $20 \mathrm{~m}$ ), plus about 80 smaller ones (75\% of them being portable), represented by about 110 APLF members, coming mainly from France, but also from Belgium, Morocco, Tunisia, Québec, Switzerland, Roumania. The APLF diffuses an annual Journal Planétariums, organises an annual Conference since 1984, produces and diffuses books and CDROMs, prepares training sessions regularly (see www. aplf-planetariums.org ).

\subsection{The APLF/ESO ALMA show for IYA: In search of our cosmic origins}

Since 2001, APLF produces planetarium shows for French and European planetariums : an unique experience! In 2001, APLF made a show on our planet as observed by satellites, in coordination with the French Space Agency CNES. In 2002-2003, for the 40 years of ESO, APLF collaborated with ESO on a show on the mysteries of the southern sky discovered thanks to the VLT. The APLF and ESO wanted to pursue this collaboration on the occasion of the IYA 2009 and came up with the idea to make a planetarium show about ALMA, the giant international project at the limit of the possible, technically and humanly.

ALMA stands for Atacama Large Millimeter/submillimeter Array, and it is without any doubt one of the largest ground-based astronomy projects of the next decade. It is presently being built on the 5,000-metres high plateau of Chajnantor in the Chilean Atacama Desert. Astronomers are now building there a fantastic facility to explore the Universe. The array of 66 antennas is fully reconfigurable and the antennas can therefore be moved from a compact configuration where all the antennas are packed in a region about 150 metres in radius, to a very wide configuration, with antennas as far as $18 \mathrm{~km}$ from the centre of the array. This provides a feature similar to the zoom of a lens camera. 


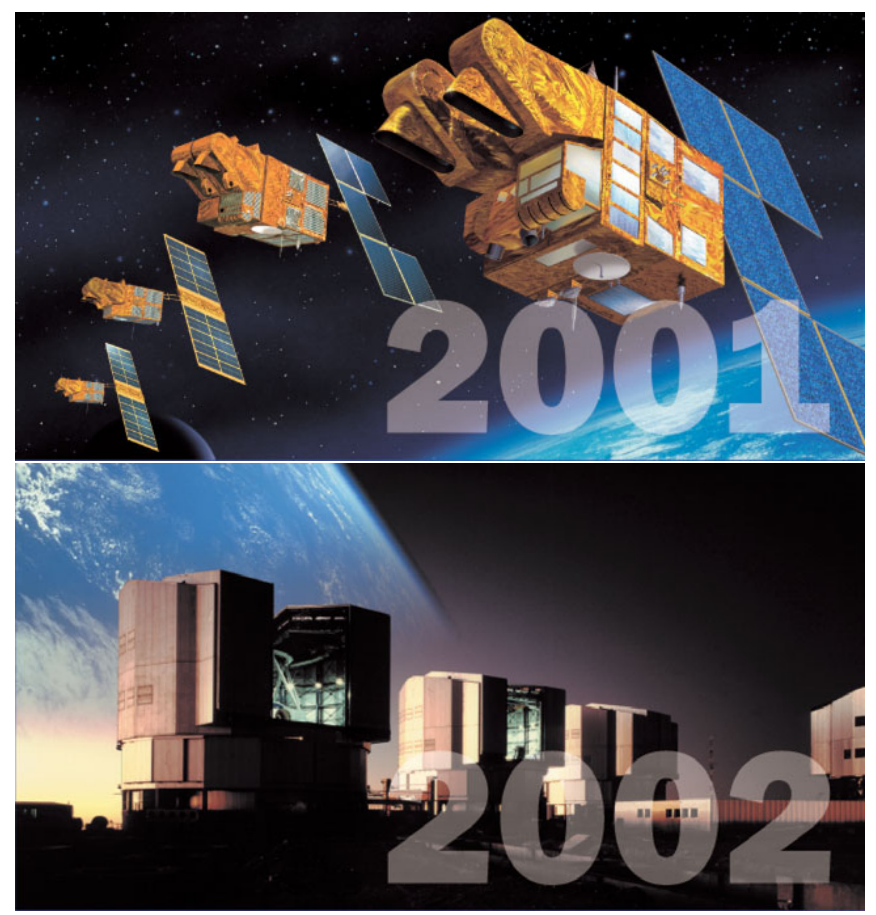

Figure 10. Associative production of shows by APLF, in partnership with the CNES (2001) and with ESO (2002)

The show is available at 3 different levels: fuldome video, AllSky immersive projection and video windows, rectangular images and videos for the smaller planetariums. The production is done by Mirage 3D. The aim of the show is to bring the most recent answers to our question : "where do we come from?" on two temporal scales : the origin of our matrix the Earth, and the origin of galaxies and the Universe. The story must be scientifically correct, working in strong interaction with specialised astronomers, avoiding naïve representations. It is not an easy task, and Robin Sip had to perform many changes to be scientifically correct. Finaly, Robin realised with patience and talent a show being attractive, bringing dream and contemplation, with musical happiness.

\section{Conclusion : what message will planetariums deliver ?}

In the beginning, celestial mechanics and he discovery of the constellations were proposed. Around 1990, multimedia prevailed, and from 1980 immersive entertainment gradually took a turn for fun and animations of Hollywood-style, with laser shows and Mickey Mouse visiting black holes. Note than an educational role more austere predominated always in Europe. Enriched by the contributions of television, film, IMAX theatres, the planetarium today visualises the universe in 3D, using powerful computers, managing terabytes to project the astronomical images transmitted by the VLT, HST, Chandra and Spitzer, but also images of geology, biology, chemistry, ... all images from current research. This theatre for all sciences issues a passport to the universe.

All scientific discoveries are sung in the planetariums, which by their technical and educational value may support the research. The audience shares the cosmic adventures, and the emotion of the discovery of splendid landscapes. Visitors are seized when they understand that the roots of humanity plunge into the history of cosmos. A universe that 


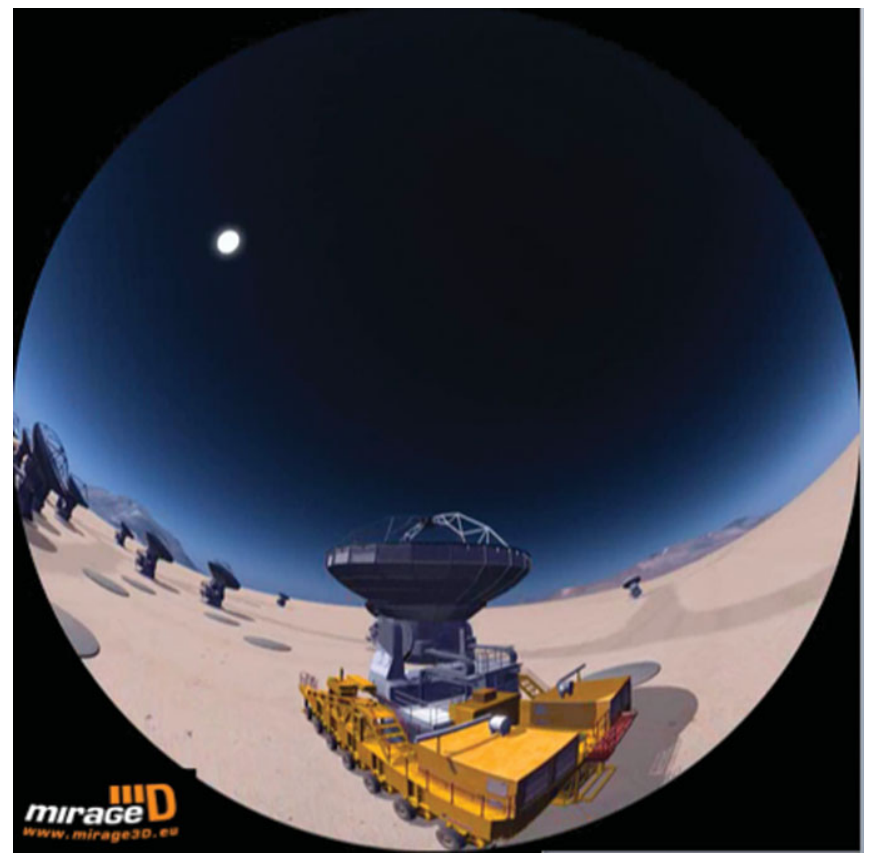

Figure 11. The ALMA transporter and telescopes - Extract of the final scene of the ALMA show dance of the antennas, with the music of Nut-Cracker from Tchaïkowski.
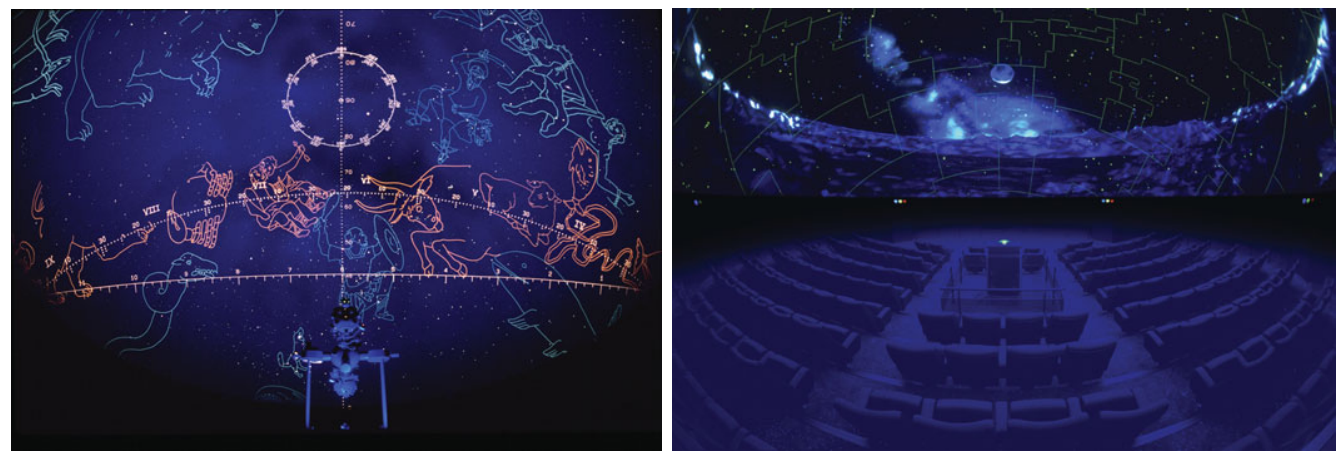

Figure 12. Digital and Optomecanical and planetariums in France: Planetarium Galilée (Evans \& Sutherland 2006) Montpellier (C)Xavier Girard) and Planetarium Ludiver (Zeiss 2006, (C)Xavier Girard).

surprises, and that invites to the dream : talking the unknown is a common concern to scientists and poets, a double identity which each Planetarium.

\section{References}

Bruno, M. 2008, Planetarian, 37, 3

Lantz, E. 2009, Planétariums, 30

Malburet, P. 2008, Planétariums, 35 